\title{
Assessment of outcomes and morbidity following diaphragmatic peritonectomy for women with ovarian carcinoma
}

\author{
Sean C. Dowdy*, Ralitsa T. Loewen, Giovanni Aletti, Simone S. Feitoza, William Cliby \\ Division of Gynecologic Surgery, Mayo Clinic, Rochester, MN 55905, USA
}

Received 29 November 2007

Available online 1 April 2008

\begin{abstract}
Objective. To describe the technique of diaphragmatic peritonectomy (DP) for ovarian cancer cytoreduction and to assess associated morbidity.

Methods. Retrospective review yielded 56 patients who underwent DP as part of a cytoreductive procedure for primary or recurrent ovarian cancer between 1988 and 2004. Patients who underwent diaphragmatic resection, removal of diaphragmatic implants with CUSA, cautery, curette, or finger fracture, and patients with pseudomyxoma were excluded from analysis.

Results. DP was performed as a component of primary or secondary cytoreduction in $37(66 \%)$ and 19 (34\%) patients, respectively. Extended procedures including bowel resection, hepatic resection, splenectomy, or radical hysterectomy were performed with DP in 47 patients ( $82 \%$ ). Resection of all disease $>1 \mathrm{~cm}$ was achieved in $95 \%$ (microscopic residuum in $43 \%$ ). For those undergoing primary cytoreduction, median survival was 59 months and 5-year survival was $49 \%$ with median follow-up of 34 months. When performed for recurrent ovarian carcinoma, 5 year survival was $16 \%$ and median survival was 23 months. No intra-operative complications could be specifically attributed to DP. Post-operative complications included a $30 \%$ rate of pleural effusion which was associated with entry into the pleural space during DP $(p<0.0001)$; thoracentesis was required in $12.5 \%$.

Conclusions. Diaphragmatic metastases are a common obstacle to optimal cytoreduction for patients with ovarian cancer. When necessary, utilizing DP in concert with other extended procedures to obtain maximal cytoreduction is associated with excellent survival. It should be recognized that DP is associated with an increased incidence of post-operative pleural effusion, particularly when the pleural space is entered. (C) 2008 Elsevier Inc. All rights reserved.
\end{abstract}

Keywords: Ovarian carcinoma; Diaphragm; Cytoreductive surgery

\section{Introduction}

Over the past decade the importance of optimal surgical cytoreduction prior to administration of cytotoxic chemotherapy has been more firmly established in the gynecologic oncology literature [1-5]. The significance of complete, as opposed to optimal, cytoreduction has also been appreciated [6]. Multiple authors have reported five-year survival rates between 40 and 50\% for patients with microscopic residual disease [1,6]. The rate of complete cytoreduction and associated survival has been shown to be both surgeon and institution dependent $[7,8]$. The radicality of procedures necessary to obtain this state of

\footnotetext{
* Corresponding author. 200 1st Street NW, Rochester, MN 55905, USA. Fax: +15072669300.

E-mail address: dowdy.sean@mayo.edu (S.C. Dowdy).
}

residuum does not appear to influence outcomes $[1,5,9]$. Although the concept of neoadjuvant chemotherapy has been championed in some American institutions [10] and in many centers in Europe [11], this practice is not currently considered standard of care for women able to tolerate surgical resection. Recently, Bristow and Chi reported a median reduction in survival of over 4 months for each cycle of pre-operative chemotherapy [12]. Thus, any improvement in post-operative morbidity afforded by neoadjuvant chemotherapy may be more than offset by a resultant diminution in oncologic outcomes.

These realizations necessitate the widespread adoption of surgical techniques that enable the surgeon to extirpate disease present not only within the pelvis, but in the upper abdomen. Thus, hepatic resection [13], splenectomy [14-16], rectosigmoid resection [17], video-assisted thoracic surgery (VATS) $[18,19]$, and diaphragmatic resection $[9,20-23]$ have been 
advocated as components of primary cytoreduction when necessary. However, it must be acknowledged that these interventions come with a potential price of increased postoperative morbidity. The purpose of this investigation was to describe the technique of diaphragmatic peritonectomy (DP), in contrast to diaphragmatic resection, and to explore potential complications unique to this procedure. This will allow readers to successfully adopt this procedure into their common practice and anticipate potential complications.

\section{Patients and methods}

From 1988 to 2004,56 patients who underwent peritoneal stripping of the diaphragm as part of a primary or secondary cytoreduction were identified from retrospective chart review. Patients who underwent diaphragmatic resection are excluded from this analysis and have been reported previously [20]. Entry into the pleural space without the need for diaphragm resection was not a reason for exclusion. In these cases the diaphragm was repaired after evacuation of the pneumothorax without the need for a chest tube. Removal of diaphragmatic implants using CUSA, cautery, curette, or finger fracture, and patients with pseudomyxoma were excluded from this analysis.

All operations were performed by a gynecologic oncologist. The decision to perform DP was dependent on the operating surgeon, the extent of disease found at laparotomy, and the condition of the patient. It has been our general practice not to perform extensive or radical procedures unless they are likely to result in an optimal cytoreduction or improve disease symptoms.

DP is performed only after the upper abdomen has been adequately exposed and the liver mobilized. Our technique is similar to that previously described for diaphragmatic resection, and we only briefly review it here [20]. A generous midline incision is extended to the level of the xiphoid process and the falciform ligament divided and incised down to the level of the hepatic veins. At this point the costal angle may be retracted cephalad using any number of retractors; we prefer to use a fixed third arm. Incision of the coronary ligament of the liver is extended to the right triangular ligament, allowing the liver to be rotated medially and inferiorly, exposing the entire diaphragm. Adequate liver mobilization is crucial, both to gain access to the diseased area, and to avoid repeated slippage of retractors along the surface of a fixed liver. This may result in a subcapsular hematoma. The right adrenal gland must also be avoided, located beneath the bare area of the liver. After the diseased area has been defined, the peritoneum is incised with a wide margin and reflected from the underlying musculature of the diaphragm (Figs. 1 and 2). This can be facilitated with the use of DeMartel clamps and sponge sticks. If significant diaphragmatic invasion is encountered, diaphragmatic resection is necessary. Otherwise, reflection of the peritoneum is continued posteriorly until the level of the coronary ligament incision is reached and the specimen removed.

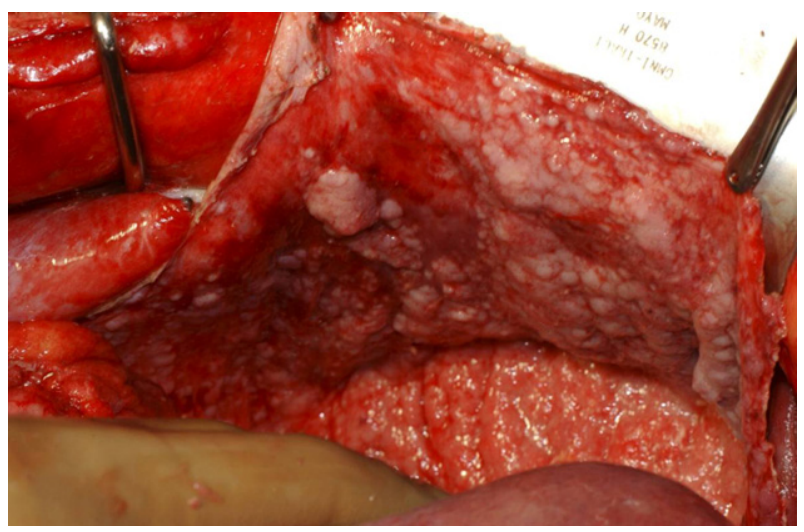

Fig. 1. Gross aspect of metastatic ovarian carcinoma to right hemidiaphragm. A Mayo-Brown retractor elevates the right costal margin over the cut edge of the diaphragmatic peritoneum. The liver has been mobilized and retracted medially.

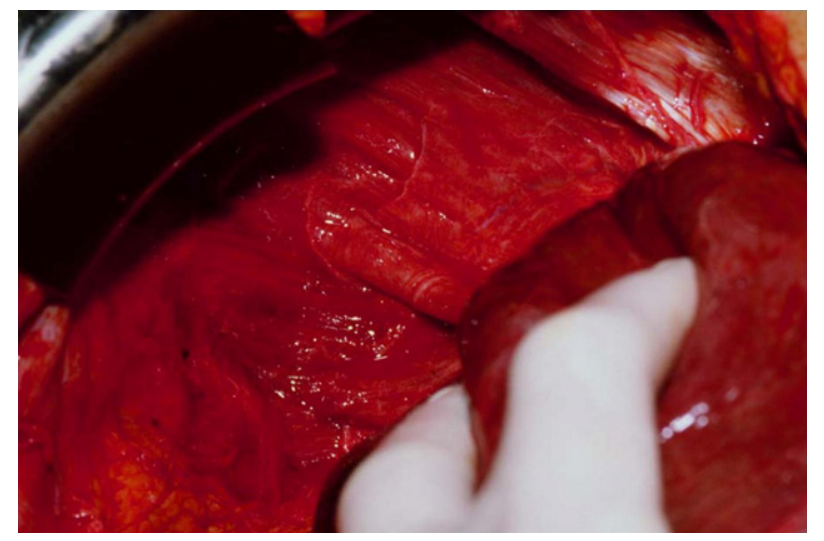

Fig. 2. Right hemidiaphragm following peritoneal stripping. The diaphragmatic musculature is seen after total resection of peritoneal disease.

Follow-up of patients was performed using information reported in clinical histories. When information about survival and recurrence was not sufficiently detailed in the histories, death certificates were obtained and letters to patients and family physicians or telephone calls were used to obtain follow-up information. We considered as censored all patients who were alive (with or without disease) at the time of follow-up or who died of a cause not related to the disease; patients who died of disease were considered uncensored.

Statistical analyses were performed with Fisher's exact tests to evaluate the relationship between pairs of categorical variables. Mann-Whitney $U$ test was used to test for differences between groups in the distributions of continuous measures. Survival curves were determined by the Kaplan-Meier product-limit method. Analyses of predictors of disease-related survival (DRS) were performed using log-rank test. Differences between groups were considered statistically significant at $p \leq 0.05$. JMP statistical program (version 4.0.4, SAS Institute, Inc., Cray, North Carolina) was used for the analysis. This investigation was approved by the Institutional Review Board of Mayo Foundation. In accordance with the Minnesota Statute for Use of Medical Information in Research, only those patients who consented to the use of their medical records were included in this analysis.

\section{Results}

Patient characteristics are shown in Table 1. Most patients had high grade, stage IIIC disease and underwent DP as part of a primary cytoreductive procedure for ovarian carcinoma. The majority of the procedures were performed only on the right hemidiaphragm, consistent with the known circulation of peritoneal fluids along the right colic gutter [4]. As recorded in Table 2, $82 \%$ of patients underwent extended procedures in addition to diaphragmatic resection, resulting in $95 \%$ of patients in this cohort having an optimal cytoreduction $(43 \%$ with microscopic residual, $52 \%$ with macroscopic disease measuring less than $1 \mathrm{~cm}$ ). All three patients with residual disease greater than $1 \mathrm{~cm}$ died from disease within 12 months.

Post-operative complications occurred in 16 patients and are listed in Table 3. The most common complication, pleural effusion, developed in 12 of 40 patients with available postoperative chest films (30\%). Thoracentesis or placement of a chest tube was required in 5 patients $(12.5 \%)$ with pleural effusions; the remaining effusions resolved with conservative management or were asymptomatic. For the purposes of comparison, we determined the incidence of post-operative thoracentesis in our ovarian cancer patients who did not 
Table 1

Patient characteristics

\begin{tabular}{lrrlr}
\hline Characteristic & $N$ & $\%$ & Median & Range \\
\hline Age & & & 56 & $21-77$ \\
Primary cytoreduction & 37 & 66 & & \\
Secondary cytoreduction & 19 & 34 & & \\
Tumor grade & & & & \\
1/2 & 2 & 4 & & \\
$3 / 4$ & 54 & 96 & \\
Stage & & & \\
IIIC & 50 & 89 & & \\
IV & 6 & 10 & \\
& & & \\
Histological subtype & & & \\
Serous & 47 & 84 & \\
Endometrioid & 4 & 7 & \\
Clear cell & 3 & 5 & \\
MMMT & 1 & 2 & \\
Immature teratoma & 1 & 2 & \\
Side of diaphragmatic stripping & & & \\
Right & 46 & 82 & \\
Bilateral & 9 & 16 & \\
Left & 1 & 2 & \\
Optimal cytoreduction* & 53 & 95 & \\
Suboptimal cytoreduction & 3 & 5 & \\
\hline
\end{tabular}

*Defined here as resection of all tumor nodules measuring greater than $1 \mathrm{~cm}$.

undergo diaphragmatic procedures over a ten year period. Thoracentesis in the peri-operative period was necessary in only 2 of 287 patients with stage IIIC disease and carcinomatosis $(0.7 \%)$ and in 4 of 95 patients with documented stage IV disease $(4.2 \%)$.

Interestingly, the only predictor for the development of postoperative pleural effusions was entry into the pleural space at the time of DP $(p<0.0001)$. In fact, entry into the pleural space occurred in all 5 patients requiring thoracentesis. For the 16 patients in whom the pleural space was entered, $10(63 \%)$ developed an effusion; 5 patients $(31 \%)$ required thoracentesis. In contrast only 2 of 40 patients $(5 \%)$ in whom the pleural space was not entered developed effusions and none required thoracentesis.

In regards to the remaining complications, the patients with pancreatitis and ileus recovered completely with conservative

Table 2

Extended procedures performed together with diaphragmatic peritonectomy

\begin{tabular}{lcr}
\hline Procedure & $N$ & $\%$ \\
\hline Large bowel resection & 29 & 52 \\
Splenectomy & 12 & 21 \\
Small bowel resection & 11 & 19 \\
Radical hysterectomy & 11 & 19 \\
Liver resection & 4 & 7 \\
Inguinal lymphadenectomy & 1 & 2 \\
Total patients undergoing extended procedures & 46 & 82 \\
$\quad$ other than diaphragmatic peritonectomy & &
\end{tabular}

Note. percentages are not additive as multiple procedures were performed on some patients.
Table 3

Complications following diaphragmatic peritonectomy

\begin{tabular}{lrr}
\hline Complication & $N$ & $\%$ \\
\hline Pleural effusion* & 12 & 30 \\
Ileus & 2 & 4 \\
Pancreatitis & 2 & 4 \\
MI & 1 & 2 \\
Pelvic abscess & 1 & 2 \\
DVT & 1 & 2 \\
Intra-operative atrial fibrillation & 1 & 2 \\
\hline
\end{tabular}

*5 patients $(12.5 \%)$ required thoracentesis or chest tube placement.

management. The patient who suffered a myocardial infarction post-operatively recovered fully. The pelvic abscess resolved after drainage under CT guidance and treatment with antibiotics. An additional patient underwent successful intraoperative cardioversion for unstable atrial fibrillation. Intra- or post-operative blood transfusion was necessary in $64 \%$ of patients; a median of $2 \mathrm{U}$ of blood were transfused. Given the high percentage of extended procedures required for cytoreduction in this cohort $(82 \%)$, it is not possible to determine the blood loss attributable specifically to DP.

Given the absence of a control group, the significance of survival data in this investigation is of questionable value. However, $\mathrm{K}-\mathrm{M}$ survival data for patients following cytoreduction for primary ovarian cancer is presented in Fig. 3. Median survival was 59 months and 5-year survival was $49 \%$ over a median follow-up of 34 months. For those patients who underwent DP for recurrent disease, 5-year survival was 16\% and median survival was an additional 23 months from the time of secondary cytoreduction. Differences in overall survival between those with primary and recurrent disease were of borderline significance $(p=0.07)$.

As we have accumulated more experience with this technique and anecdotally noted acceptable morbidity, the use of DP has been utilized at our institution more frequently in recent years. DP was performed in 23 patients in the five-year period between 2000 and 2004 as compared to 30 patients between 1995 and 1999, a 30\% increase. This procedure also occurred more commonly at primary cytoreductive procedures:

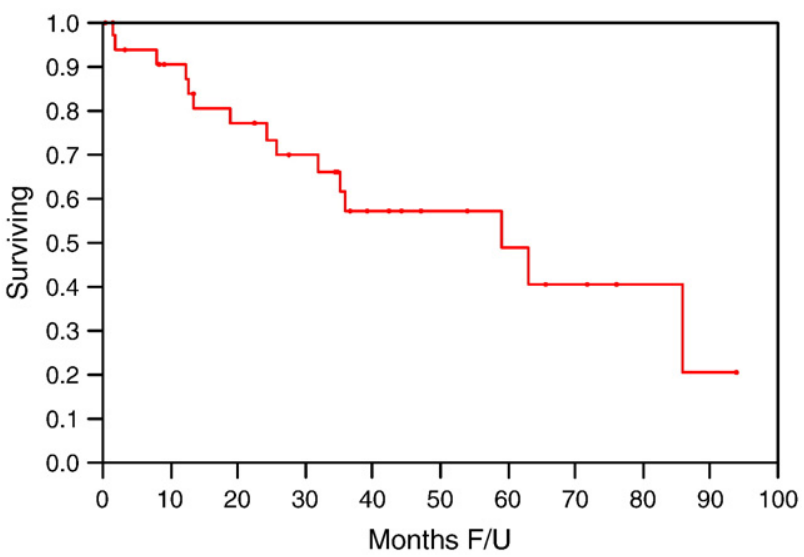

Fig. 3. Kaplan-Meier survival following diaphragmatic peritonectomy for patients with primary ovarian cancer. 
in the most recent 5 -year period $73 \%$ occurred in the primary setting vs. 57\% between 1995 and 1999. In a recent internal quality assessment of ovarian cancer surgery at our institution, we noted an increase in the utilization of all diaphragmatic procedures at primary surgery from $23 \%$ of cases with diaphragm involvement in the period between 1994 and 1998 to $59 \%$ in 2006 (unpublished, internal data) [24].

\section{Discussion}

The clear association between optimal cytoreduction and improved survival in patients with advanced ovarian cancer has resulted in the adoption of techniques to address upper abdominal disease in many centers [25]. We and others have provided evidence that survival benefits are not compromised when radical procedures, including diaphragm resection, are necessary to achieve optimal cytoreduction $[1,21,26]$. In a survey of the SGO, $76 \%$ of members polled stated that diaphragmatic disease was a barrier to optimal cytoreduction [27]. In the current investigation we analyzed data from patients who underwent DP, excluding from analysis those patients who underwent diaphragmatic resection, in order to describe the technique and identify unique complications or signs of potential patient compromise. The cohort of patients undergoing diaphragmatic resection has been analyzed previously [20].

As described here, DP is a relatively simple procedure and greatly facilitated by hepatic mobilization. No intra-operative complications were attributed to DP. Even when performed in concert with other extended procedures ( $82 \%$ in this study) DP was well-tolerated. The one exception to this is the relatively high incidence of post-operative pleural effusions, $30 \%$ in our series. While difficult to compare without a proper control group, patients having DP required thoracentesis at a rate significantly higher than that of historical controls at our institution that underwent surgical cytoreduction without diaphragmatic surgery ( $12.5 \%$ vs. 0.7 and $4.2 \%$ for stage IIIC with carcinomatosis and stage IV, respectively). In the only other report of DP which included such information, Eisenhauer reported an ipsilateral effusion rate of $60 \%$ among 40 patients without pre-operative effusion [28]. However, this series of 59 patients included both diaphragmatic resection and DP. Postoperative thoracentesis or chest tube placement was required in $15 \%$ of patients. This rate of intervention is similar to our observed rate of $12.5 \%$. Comparison of this rate is more legitimate recognizing that the overall rate of pleural effusions (including asymptomatic effusions) will be dependent on the threshold of the surgical service for obtaining chest films in the post-operative period.

Using multivariate analysis, Eisenhauer et al. found that only hepatic mobilization was a statistically significant predictor of pleural effusions. Since we routinely divide the hepatic ligaments in order to mobilize the liver, we were unable to test this association in our cohort. We feel that hepatic mobilization is critical to attain adequate exposure and to perform a safe and complete resection, and outweighs a potential increase in the rate of pleural effusions. Furthermore, this association is likely to be spurious given that all our patients had liver mobilization, but the rate of thoracentesis was nearly identical to that reported by Eisenhauer et al. In contrast, we found a very strong association between diaphragmatic perforation and post-operative pleural effusion $(p<0.0001)$. When the pleural space was entered, $63 \%$ developed an effusion and $31 \%$ required thoracentesis. Although we routinely check the diaphragm for air leaks after repair, it may be that with continued respiration ascites are drawn into the pleural space, resulting in an effusion in the post-operative period.

Although we concur with Eisenhauer et al. that the relatively low rate of requirement for thoracentesis does not support the routine use of prophylactic interventions such as chest tube placement, the respiratory status of patients with diaphragmatic perforation at the time of DP should be observed carefully in the post-operative period. Otherwise, the rate and type of complications observed was consistent with that seen after aggressive cytoreduction without DP. We currently utilize this procedure at more than twice the rate of a decade ago for patients with primary ovarian cancer, and as noted in other practices have witnessed a resultant increase in optimal cytoreduction rates [25].

While the purpose of this investigation was to describe the technique of DP and evaluate associated complications, we also note that survival for these patients was excellent. For patients with primary ovarian cancer, the 5-year survival was $49 \%$. We have shown previously that in patients with diaphragmatic disease, diaphragmatic resection or peritonectomy was associated with improved survival, even when the analysis was limited to those patients optimally cytoreduced (55 vs. $28 \% 5$ year survival, $p<0.001$ ) [21]. In this report three patients underwent DP yet were left with a suboptimal cytoreduction. It is not our general practice to perform extended procedures such as DP in the context of a suboptimal cytoreduction. Rarely, however, initial assessment of cytoreducibility is incorrect.

In summary, DP is a relatively straightforward procedure that should be a part of the skill set of any surgeon performing surgical cytoreduction for patients with ovarian cancer. Adoption of this technique has the potential to improve maximal cytoreduction rates and patient outcomes. It should be noted that these patients appear to be more prone to the development of post-operative pleural effusions. While no prophylactic intervention is necessary, due vigilance is required to assess these patients for shortness of breath and the possible need for thoracentesis.

\section{References}

[1] Aletti GD, Dowdy SC, Gostout BS, Jones MB, Stanhope CR, Wilson TO, et al. Aggressive surgical effort and improved survival in advanced-stage ovarian cancer. Obstet Gynecol 2006;107:77-85.

[2] Bristow RE, Tomacruz RS, Armstrong DK, Trimble EL, Montz FJ. Survival effect of maximal cytoreductive surgery for advanced ovarian carcinoma during the platinum era: a meta-analysis. J Clin Oncol 2002;20: 1248-59.

[3] Griffiths CT. Surgical resection of tumor bulk in the primary treatment of ovarian carcinoma. Natl Cancer Inst Monogr 1975;42:101-4.

[4] Ulfelder H, Meigs JV. The surgery of advanced pelvic cancer in women. N Engl J Med 1952;246:243-7. 
[5] Eisenkop SM, Spirtos NM, Friedman RL, Lin WC, Pisani AL, Perticucci S. Relative influences of tumor volume before surgery and the cytoreductive outcome on survival for patients with advanced ovarian cancer: a prospective study. Gynecol Oncol 2003;90:390-6.

[6] Eisenkop SM, Friedman RL, Wang HJ. Complete cytoreductive surgery is feasible and maximizes survival in patients with advanced epithelial ovarian cancer: a prospective study. Gynecol Oncol 1998;69: $103-8$.

[7] Aletti GD, Gostout BS, Podratz KC, Cliby WA. Ovarian cancer surgical resectability: relative impact of disease, patient status, and surgeon. Gynecol Oncol 2006;100:33-7.

[8] Earle CC, Schrag D, Neville BA, Yabroff KR, Topor M, Fahey A, et al. Effect of surgeon specialty on processes of care and outcomes for ovarian cancer patients. J Natl Cancer Inst 2006;98:172-80.

[9] Eisenhauer EL, Abu-Rustum NR, Sonoda Y, Levine DA, Poynor EA, Aghajanian $\mathrm{C}$, et al. The addition of extensive upper abdominal surgery to achieve optimal cytoreduction improves survival in patients with stages IIIC-IV epithelial ovarian cancer. Gynecol Oncol 2006;103: 1083-90.

[10] Schwartz PE, Chambers JT, Makuch R. Neoadjuvant chemotherapy for advanced ovarian cancer. Gynecol Oncol 1994;53:33-7.

[11] Ansquer Y, Leblanc E, Clough K, Morice P, Dauplat J, Mathevet P, et al. Neoadjuvant chemotherapy for unresectable ovarian carcinoma: a French multicenter study. Cancer 2001;91:2329-34.

[12] Bristow RE, Chi DS. Platinum-based neoadjuvant chemotherapy and interval surgical cytoreduction for advanced ovarian cancer: a metaanalysis. Gynecol Oncol 2006;103:1070-6.

[13] Merideth MA, Cliby WA, Keeney GL, Lesnick TG, Nagorney DM, Podratz KC. Hepatic resection for metachronous metastases from ovarian carcinoma. Gynecol Oncol 2003;89:16-21.

[14] Chen LM, Leuchter RS, Lagasse LD, Karlan BY. Splenectomy and surgical cytoreduction for ovarian cancer. Gynecol Oncol 2000;77: $362-8$.

[15] Eisenkop SM, Spirtos NM, Lin WC. Splenectomy in the context of primary cytoreductive operations for advanced epithelial ovarian cancer. Gynecol Oncol 2006;100:344-8.

[16] Magtibay PM, Adams PB, Silverman MB, Cha SS, Podratz KC. Splenectomy as part of cytoreductive surgery in ovarian cancer. Gynecol Oncol 2006;102:369-74.
[17] Aletti GD, Podratz KC, Jones MB, Cliby WA. Role of rectosigmoidectomy and stripping of pelvic peritoneum in outcomes of patients with advanced ovarian cancer. J Am Coll Surg 2006;203:521-6.

[18] Eisenkop SM. Thoracoscopy for the management of advanced epithelial ovarian cancer - a preliminary report. Gynecol Oncol 2002;84:315-20.

[19] Juretzka MM, Abu-Rustum NR, Sonoda Y, Downey RJ, Flores RM, Park BJ, et al. The impact of video-assisted thoracic surgery (VATS) in patients with suspected advanced ovarian malignancies and pleural effusions. Gynecol Oncol 2007;104:670-4.

[20] Cliby W, Dowdy S, Feitoza SS, Gostout BS, Podratz KC. Diaphragm resection for ovarian cancer: technique and short-term complications. Gynecol Oncol 2004;94:655-60.

[21] Aletti GD, Dowdy SC, Podratz KC, Cliby WA. Surgical treatment of diaphragm disease correlates with improved survival in optimally debulked advanced stage ovarian cancer. Gynecol Oncol 2006;100:283-7.

[22] Montz FJ, Schlaerth JB, Berek JS. Resection of diaphragmatic peritoneum and muscle: role in cytoreductive surgery for ovarian cancer. Gynecol Oncol 1989;35:338-40.

[23] Silver DF. Full-thickness diaphragmatic resection with simple and secure closure to accomplish complete cytoreductive surgery for patients with ovarian cancer. Gynecol Oncol 2004;95:384-7.

[24] Aletti GD, Dowdy SC, Gostout BS, Jones MB, Stanhope RC, Wilson TO, et al. Quality improvement (QI) in the surgical approach to advanced ovarian cancer: Mayo clinic experience. Presented at the Central Association of Obstetricians and Gynecologists, Chicago, IL; 2007.

[25] Chi DS, Franklin CC, Levine DA, Akselrod F, Sabbatini P, Jarnagin WR, et al. Improved optimal cytoreduction rates for stages IIIC and IV epithelial ovarian, fallopian tube, and primary peritoneal cancer: a change in surgical approach. Gynecol Oncol 2004;94:650-4.

[26] Eisenkop SM, Spirtos NM. Procedures required to accomplish complete cytoreduction of ovarian cancer: is there a correlation with "biological aggressiveness" and survival? Gynecol Oncol 2001;82:435-41.

[27] Eisenkop SM, Spirtos NM. What are the current surgical objectives, strategies, and technical capabilities of gynecologic oncologists treating advanced epithelial ovarian cancer? Gynecol Oncol 2001;82:489-97.

[28] Eisenhauer EL, D’Angelica MI, Abu-Rustum NR, Sonoda Y, Jarnagin WR, Barakat RR, et al. Incidence and management of pleural effusions after diaphragm peritonectomy or resection for advanced mullerian cancer. Gynecol Oncol 2006;103:871-7. 